\title{
Electrochemical Capacitance and Ionic Transport in the Mesoporous Shell of a Hierarchical Porous Core-Shell Carbon Structure
}

\author{
Fujun Li, Madeleine Morris, Kwong-Yu Chan* \\ Received (in $X X X, X X X)$ Xth $X X X X X X X X X 200 X$, Accepted Xth $X X X X X X X X X 200 X$ \\ First published on the web Xth $X X X X X X X X X 200 X$ \\ DOI: 10.1039/b000000x
}

A three-dimentional (3D) hierarchical porous carbon structure was prepared with possible variations of porositity at three levels of length scales. The carbon structure was template-synthesized from a core-shell silica sphere assembly. The assynthesized carbon featured an semi-ordered porous structure with hollow macro-cores $(330 \mathrm{~nm})$ surrounded by a

10 mesoporous shell containing uniform pores of $3.9 \mathrm{~nm}$ and distinct interstitial space between the core-shell domains. The mesoporous shell thickness was stepwise increased from 0, 25, 50 to $100 \mathrm{~nm}$ while keeping an identical core size to create a family of hierarcical porous structures for a systematic investigation of electrochemical capacitance and ionic transport. The shell thickness affected the overall porosity and relative porosities of the shell, core, and interstitial regions. A thicker mesoporous shell possessed a higher surface area which led to a proportional increase in 15 electrochemical capacitance which can be fully realised at low scan rates. For the carbon structure with the maximum shell thickness of $100 \mathrm{~nm}$, electrochemical capacitance per unit area and power density declined at high scan rates and high currents when ionic transport through long mesopores became limiting. The power density of the better assynthesized porous carbon was up to $11.7 \mathrm{~kW} \mathrm{~kg}^{-1}$ when the corersponding energy density was $5.9 \mathrm{Wh} \mathrm{kg}^{-1}$.

\section{Introduction}

20 An electrical double-layer capacitor (EDLC) stores and releases electric energy through the transfer of ions to and from the electrode/electrolyte interface, without a Faradic reaction. The behavior of EDLC has attracted considerable research interests in recent years. ${ }^{1-3}$ The capacitance of EDL 25 is related to the ion accessible surface area, the larger of which leads to higher capacitance and higher energy density. Micropores (less than $2 \mathrm{~nm}$ ) normally yield higher surface area on gravimetric or volumetric basis compared to mesopores and macropores. ${ }^{3}$ However, very small micropores, 30 e.g. less than $1 \mathrm{~nm}$ are not easily accessible by ions. ${ }^{4}$ How fast the ions move within the porous sturcuture will determine the power density of EDLC, ${ }^{5,6}$ and the micropores were found to have more resistance to fast ion transportation. A trade-off is needed between high power density which depends on large 35 pores and energy density which depends on surface area. Therefore, designing the pore size distribution and the rational texture in electrode materials are critical to optimize electrical capacitance performance.

Three-dimentional (3D) hierarchical porous carbons have 40 been proposed to yield good specific energy density and power density. ${ }^{7-10}$ It is desirable to structure the porous carbon with pores of multiple length scales and with micropores/mesopores interconnected by larger pores. An optimum structure is believed to possess high surface smaller 45 pores interconnected with larger sets of pores with efficient ion diffusion pathways. The particle size of carbide-derived carbons (CDCs) has been shown to affect the pore accessibility for ion migration and adsorption and can limit the EDL capacitance. ${ }^{11}$ Moriguchi et al. ${ }^{8}$ and Yamada et al. ${ }^{9}$ 50 reported a specific capacitance of $20 \mathrm{~F} \mathrm{~cm}^{-2}$ in a porous carbon with a $3 \mathrm{D}$ periodic structure containing mainly mesopores and macropores. Wang et al. ${ }^{10}$ reported that high- rate performance of hierarchical porous carbon could be attributed to a combination of macroporous cores and a 55 mesoporous wall while the carbon texture was not ordered. Comparing a series of similarly prepared porous carbon structures templated from KIT mesoporous silica, Li et al. ${ }^{12}$ showed that additional larger pores interconnecting mesopores can provide more effective ion diffusion and 60 enhance high-rate capacitance performance.

Three-dimensional ordered porous carbon strcutures were synthesized via templating an aseembly of $\mathrm{SiO}_{2}$ or polystyrene solid spheres and their EDLC studied ${ }^{8,9,13,14}$. Most of these investigations seeked performance in hierarchical porous 65 structures without stepwise variations of structural parameters. It is desirable to investigate systematically the contribution to capacitance and ionic transport limitation in each length scale of the porosity hierachy.

A hierarchical porous carbon structure synthesized from an 70 assembly of macrospheres a few hundred nanometers in size with variable mesoporous shell thickness is proposed here as shown in Fig. 1. This simple structure has pores at several

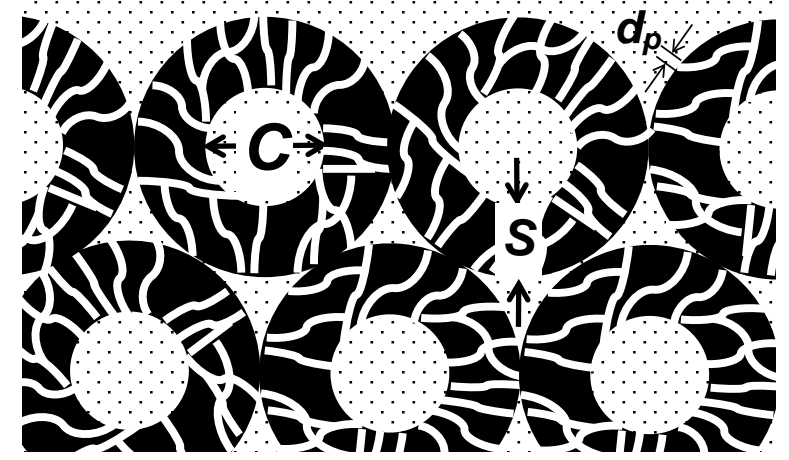

Fig. 1. A core-mesoporous shell structure with three variable 75 geometric parameters $d_{p}, S$, and $C$ controlling different levels of the hierarchical structure. 
length scales distributed over the hollow cores, mesoporous shell, and the interstitial regions. The "hollow coremesoporous shell" carbon structure can be templatesynthesized from solid silica spheres each coated with a 5 mesoporous silica shell. The three structural parameters: the mesopore diamter, $d_{p}$; mesoporous shell thickness, $S$; and macropore diameter, $\mathrm{C}$ can be separately tuned and controlled in the synthesis. This provides a very useful model structure for studying ionic transport and surface limited 10 electrochemical reactions in relation to porosities at different length scales. A variation to the structure in Fig. 1 has been reported by $\mathrm{Yu}$ and co-workers ${ }^{13,14}$ who synthesized an ordered assembly of hollow cores with mesoporous interstitial regions in the limiting case of $S=0$. The mesopores were 15 created in the interstital regions by templating small silica spheres $^{13,14}$. Their structure has the mesoporous interstitial regions inter-connected by thin areas of the $S=0$ limit and leads to poor connectivity and high ohmic resistance in the overall structure.

20

We report here the synthesis and electrochemical investigations of the hierachical structure with a focus on the role of the mesoporous shell region in the overall capacitance and ionic transport. A mesoporous shell of tens of nanometers 25 thick $(S)$ with uniform pores typically $3.9 \mathrm{~nm}\left(d_{p}\right)$ surrounding hollow cores of $330 \mathrm{~nm}(C)$ was created in the hierarchical carbon strucutre. The mesoporous shell thickness was stepwise increased from $0,25,50$ to $100 \mathrm{~nm}$. The hierarcical porous carbon was studied as an EDLC material. The effects 30 and impacts of shell thickness on electrochemical capacitance and ionic transport were discussed with consideration of the relative contributions of different regions: the hollow core, the mesoporous shell and the interstitial regions.

\section{Experimental}

\section{Preparation of core-shell silicas}

$\mathrm{SiO}_{2}$ solid spheres were prepared by the Stöber method ${ }^{15} \cdot 7.1$ $\mathrm{ml}$ concentrated ammonium solution was stirred into a mixture of $178.6 \mathrm{ml}$ ethanol and $14.3 \mathrm{ml}$ water for $1 \mathrm{hr}$. Then $10.7 \mathrm{ml}$ tetraethoxysilicate (TEOS) was added and stirred for 40 another 6 hrs. The product was collected to be $\mathrm{SiO}_{2}$ solid spheres with a diameter of $350 \mathrm{~nm}$. To add a meosporous silica shell to the silica spheres, a second step $^{16}$ synthesis proceeds as follows. A solution of $2.4 \mathrm{~g}$ cetyltrimethylammonium bromide (CTAB) in $40 \mathrm{ml}$ water and ${ }_{45} 20 \mathrm{ml}$ ethanol was mixed into the above mixture and kept stirring for 12 hrs. Then $4.1 \mathrm{ml}$ TEOS was introduced and after another $12 \mathrm{hrs}$, the as-synthesized product was filtered and treated at $500{ }^{\circ} \mathrm{C}$ in air for 3 hrs to be core-shell silica with a thickness of $50 \mathrm{~nm}$. Using the above procedure, two 50 other sets of core-shell $\mathrm{SiO}_{2}$ particles with thickness of 80 and $150 \mathrm{~nm}$ were prepared via a layer-by-layer technique. The assynthesized $\mathrm{SiO}_{2}$ products with different shell thickness were denoted as CS0, CS50, CS80 and CS150 corresponding to 0, 50, 80 and $150 \mathrm{~nm}$ thick shells of the silica particles.

${ }_{55}$ Preparation of porous carbons
One gram of $\mathrm{SiO}_{2}$ spheres were dispersed with ultrasound in $20 \mathrm{ml}$ ethanol aqueous solution $(1: 1, \mathrm{v} / \mathrm{v})$. The mixture was dried at $45{ }^{\circ} \mathrm{C}$ to get a $\mathrm{SiO}_{2}$ assembly and then calcined at $750^{\circ} \mathrm{C}$ in air for 2 hrs to increase contact between $\mathrm{SiO}_{2}$ 60 spheres. After cooling, the $\mathrm{SiO}_{2}$ assembly was filled with a solution of furfuryl alcohol (FA) carbon precursor, and oxalic acid as catalyst with a molar ratio of $30: 1$. The as-synthesized composites were kept at $80{ }^{\circ} \mathrm{C}$ and then $160{ }^{\circ} \mathrm{C}$ for 3 hrs in air, then carbonized in a tube furnace at $900{ }^{\circ} \mathrm{C}$ for 5 hrs under an 65 argon atmosphere. Finally, the $\mathrm{SiO}_{2}$ templates were etched from the carbon/silica composites using $10 \mathrm{wt} \%$ hydrofluoric acid (HF). The product 3D porous carbons were washed thoroughly with water and ethanol several times and dried at $60{ }^{\circ} \mathrm{C}$ in a vacuum oven overnight. The as-synthesized porous 70 carbons from the templates CS0, CS50 CS80 and CS150 assemblies were denoted as C-CS0, C-CS50 C-CS80 and CCS150, respectively. All the carbon samples have no silica residuals remained as analyzed by energy dispersive $\mathrm{x}$-ray spectroscopy (EDX).

\section{Characterization}

Scanning transition electron microscopy (STEM, Philips TECNAI 20 with $200 \mathrm{kV}$ accelerating voltage) and field emission scanning electron microscopy (SEM, Hitachi S4800) were used to characterize the structures of the as80 synthesized porous carbons. Nitrogen sorption isotherms were obtained on a Micromeritics ASAP 2020 analyzer at $77 \mathrm{~K}$. The surface area was determined by using the BrunauerEmmett-Teller (BET) method and the pore size distribution $(\mathrm{BJH})$ was calculated via the Barrett-Joyner-Halenda $(\mathrm{BJH})$ 85 method. The pore volume was integrated from the BJH pore size distribution (PSD) curves.

\section{Electrochemical measurements}

Working electrodes were prepared by pressing a slurry comprising $95 \mathrm{wt} \%$ porous carbons and $5 \mathrm{wt} \%$ 90 poly(tetrafluoroethylene) PTFE dispersed in pure ethanol, onto $1 \mathrm{x} 1 \mathrm{~cm}^{2}$ nickel foam. The mass of porous carbon loaded on each of the working electrodes was $5.0 \mathrm{mg}$. Before testing, the working electrodes were immersed in $6.0 \mathrm{M} \mathrm{KOH}$ solution overnight. All the electrochemical tests were obtained on ${ }_{95}$ Solartron 1287 using a three-electrode cell. A Pt plate was applied as the counter electrode and a $\mathrm{Hg} / \mathrm{HgO}$ electrode in 6.0 M KOH solution was the reference electrode. The capacitance was integrated from cyclic voltammetries (CVs) at various scan rates within a voltage window between 0 and $1000.9 \mathrm{~V}$ vs. $\mathrm{Hg} / \mathrm{HgO}$ and the charge/discharge processes were also conducted within the same voltage window at different current loadings.

\section{Results and discussions}

\section{Synthesized Structures}

105 TEM images of silica templates with different shell thicknesses are shown in Fig. 2. Silica solid spheres CS0 in Fig. 2(a) have a diameter estimated to be $350 \mathrm{~nm}$. CS50, CS80 and CS150 in Figs. 2(b), (c) and (d) have core the same size as CS0. Shells with thickness of 50, 80, and $150 \mathrm{~nm}$ are 
observed in CS50 CS80 and CS150, respectively, as created by adjusting the amounts of CTAB and TEOS in the second step of the synthesis. A highly porous shell of CS150 can be observed in Fig 2(d). The silicas CS50, CS80 and CS150 were 5 analyzed by nitrogen sorption to have a sharp pore size distribution of $3.8 \mathrm{~nm}$, typical of CTAB templated materials. Silica spheres were assembled together via a slow evaporation process to give an ordered structure as a hexagonal pattern in Fig. 3.
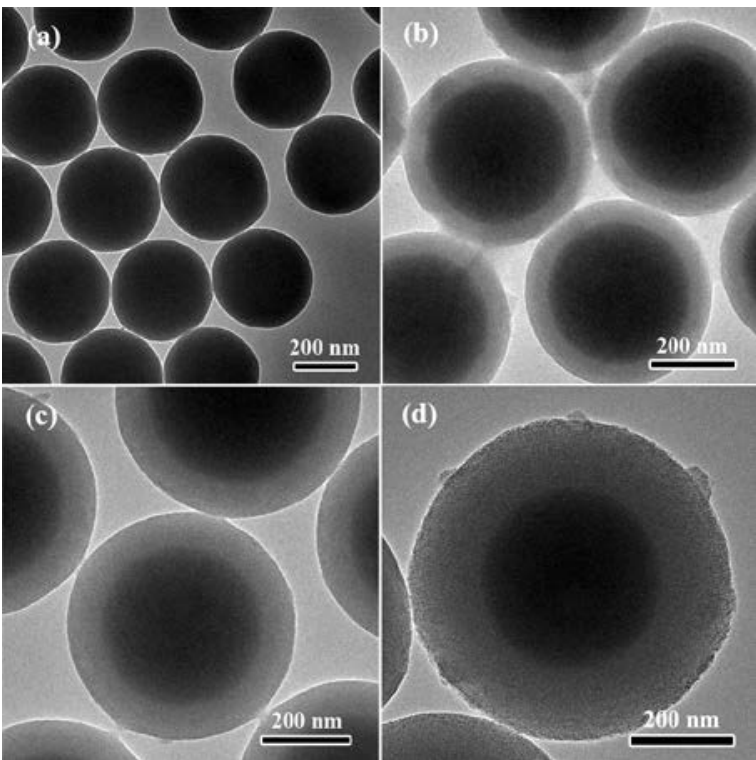

Fig. 2 TEM images of (a) CS0, (b) CS50, (c) CS80, and (d) CS150.

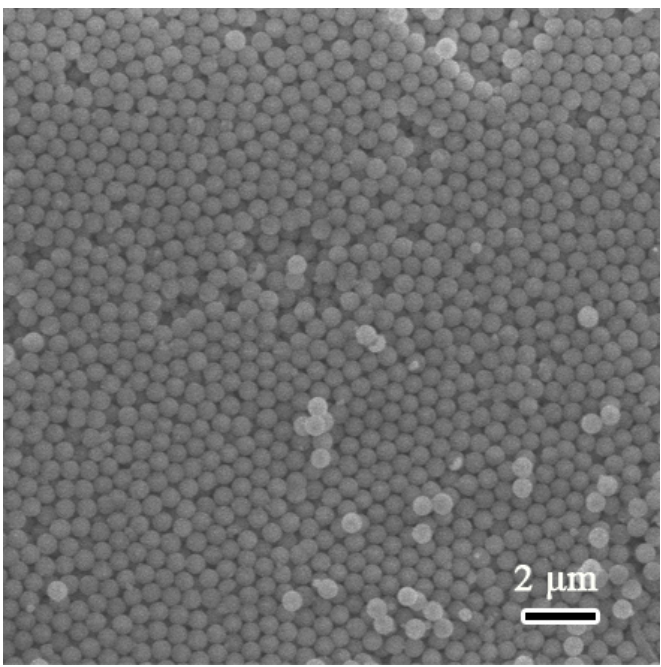

Fig. 3. SEM image of the assembly of CS150.

After removing the $\mathrm{SiO}_{2}$ template of the synthesized 15 carbon-silica composites, the carbon replicas C-CS0, C-CS50, C-CS80 and C-CS150 are imaged by SEM presented in Fig. 4. As shown in Fig. 4(a), C-CSO has an ordered porous carbon structure. Packed in a hexagonal pattern representing the [111] section of a face-centred-cubic (fcc) packing, the hollow 20 macro-cores are ca. $330 \mathrm{~nm}$ in size. The carbon structure shrunk slightly from the corresponding slica template. The contacting boundary between hollow macro-cores is very thin. This contact region and the interstitial appear porous, as shown in Fig. 4(b). The pores in the interstital region are not 25 uniform in size and could not be created by a structure directing agent since no surfactant was used in the syntheis of CS0. They were created by the thinly wetted FA precursor which carbonized without templating to yield non-uniform micropores.
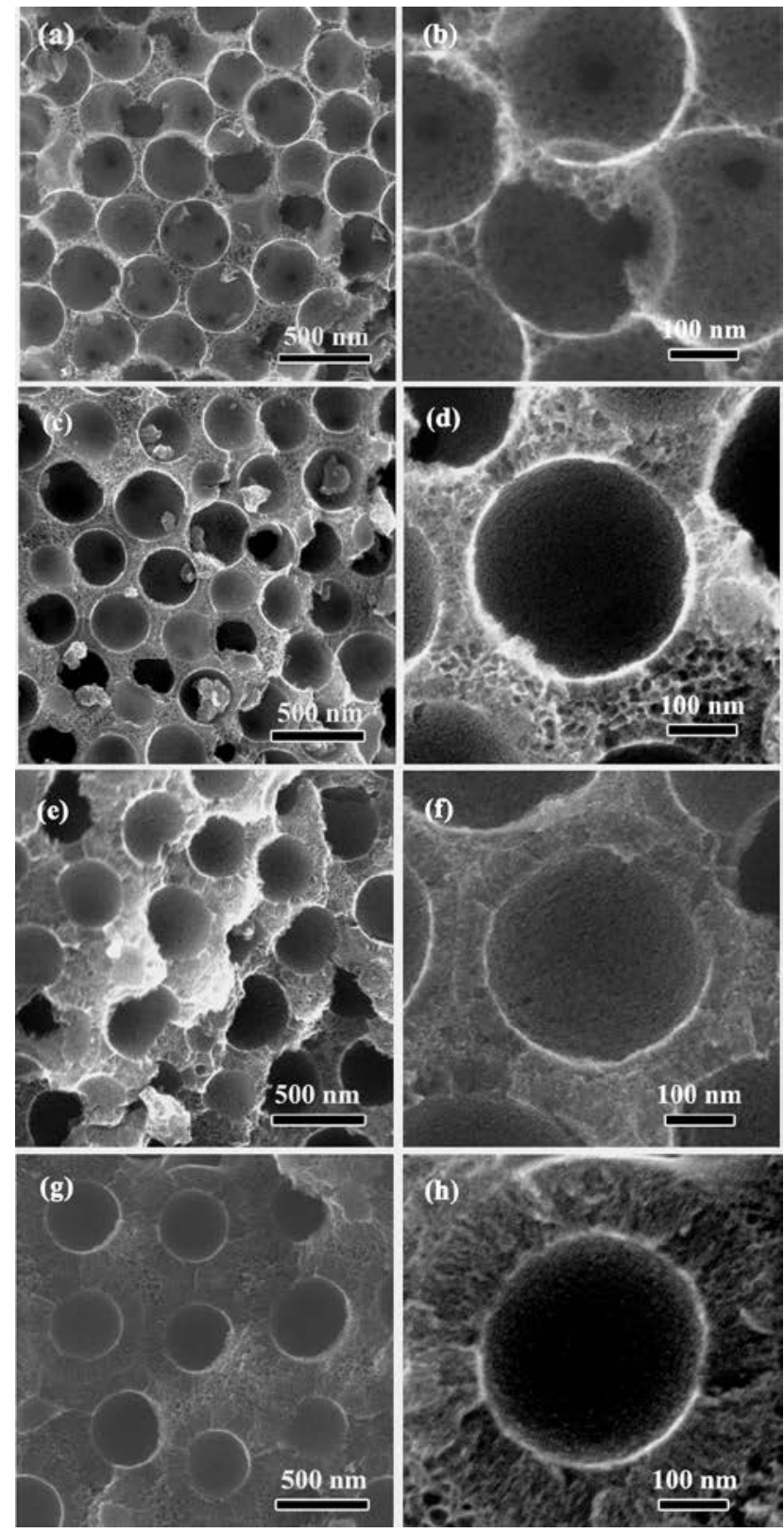

Fig. 4. SEM images of (a, b) C-CS0, (c, d) C-CS50, (e, f) C-CS80, and (g, h) C-CS150.

When the templates were changed to the core-shell silicas, the ordered porous carbon structure was also observed, as 35 shown in Figs. 4(c), 4(e), and 4(g) for C-CS50, C-CS80 and C-CS150, resepectively. The size of the hollow cores is ca. $330 \mathrm{~nm}$, the same in the four carbon structures C-CSO, CCS50, C-CS80 and C-CS150. The interstitial region can be differentiated from the shell region which is denser and 40 contains more uniform pores, as shown in Figs. 4(d), 4(f), and 4(h). After removing the silica templates, the shell thickness 
in C-CS50, C-CS80 and C-CS150 shrunk from 50, 80, and $150 \mathrm{~nm}$ of the templates to ca. 25, 50, and $100 \mathrm{~nm}$, respectively.

The carbon structures C-CS0, C-CS50, C-CS80 and C${ }_{5}$ CS150 were further characterized by $\mathrm{N}_{2}$ sorption. Their $\mathrm{N}_{2}$ soprtion isotherms at $77 \mathrm{~K}$ and pore size distributions (PSDs) according to the BJH method are presented in Fig. 5(a) and 5(b), respectively. Three typical regions in the isotherms in Fig. 5(a) can be found in all porous carbons: (i) at low relative 10 pressure the $\mathrm{N}_{2}$ volume adsorbed doesn't increase steeply, suggesting negligible amount of micropores in the synthesized porous carbons; (ii) at medium relative pressure region the desorption hysteresis indicates the existence of mesopores, agreeing with the SEM observation of mesoporous shells in 15 Fig. 4; and (iii) at relative pressure near 1.0 almost vertical tails are ascribed to macropores, shown as hollow macro-cores in Fig. 4. In particular, C-CS0 shows a broad desorption hysteresis in Fig. 5(a), maybe due to a very thin shell thickness. The hollow macro-cores are larger than $200 \mathrm{~nm}$, 20 cannot be characterized by gas sorption and do not appear in the PSDs. The PSD of C-CS0 is broad without any typical peaks. The pores of C-CS0 were created by the release of gases $\left(\mathrm{H}_{2} \mathrm{O}, \mathrm{CO}, \mathrm{CH}_{4}, \mathrm{CO}_{2}\right)$ in the carbonization process of poly(furfuryl alcohol) ${ }^{17}$ in the interstitial spaces between $\mathrm{SiO}_{2}$ 25 solid spheres templates. All C-CS50, C-CS80 and C-CS150, have similar PSDs with a sharp peak at $3.9 \mathrm{~nm}$. These $3.9 \mathrm{~nm}$ mesopores can only be present in the shell region, which is templated from the mesoporous shell of the silica templates CS50, CS80 and CS150. The pores in the mesoporous silica 30 shell are uniform and are created from the CTAB surfactant during synthesis of the silica shell. Apart from the $3.9 \mathrm{~nm}$ peak, C-CS50 C-CS80 and C-CS150 have a broad shoulder in the PSDs toward the micropores region. This branch of their PSDs is similar to the C-CS0 PSD and likely contributed by 35 the interstitial regions. The BET specific surface areas of CCS50, C-CS80 and C-CS150 are 996, 1097 and $1269 \mathrm{~m}^{2} \mathrm{~g}^{-1}$, respectively. These values are much larger than the value of C-CS0, $592 \mathrm{~m}^{2} \mathrm{~g}^{-1}$, suggesting major contribution of shell regions to surface area.
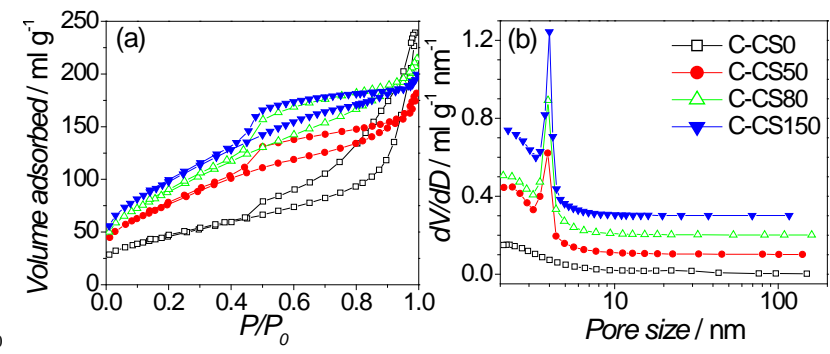

Fig. 5. $\mathrm{N}_{2}$ sorption isotherms (a) and PSDs (b) of C-CS0, C-CS50, CCS80 and C-CS150 based on BJH method.

From the SEM images, it is clear that the cores of all the carbon structures are empty and have no surface area to 45 contribute to electrochemical capacitance. To aid discussion of relative contributions of different regions to electrochemical capacitance, we list the structural details of the four carbon structures in Table 1, according to the nitrogen sorption and SEM information. The hollow macro-
50 cores are assumed to be in a fcc pattern so that the interstitial volume can be calculated. The calculations of structural parameters of the carbons are shown in electronic supporting information. For comparison, a typical synthetic ordered mesoporous carbon, CMK-3 was synthesized with properties 55 listed in Table 1.

Table 1 Structural parameters of the as-synthesized core-shell carbons.

\begin{tabular}{|c|c|c|c|c|c|}
\hline & $\begin{array}{l}\mathrm{C}- \\
\mathrm{CS} 0\end{array}$ & $\begin{array}{l}\text { C- } \\
\text { CS50 }\end{array}$ & $\begin{array}{l}\text { C- } \\
\text { CS80 }\end{array}$ & $\begin{array}{c}\text { C- } \\
\text { CS150 }\end{array}$ & $\begin{array}{l}\text { CMK } \\
-3\end{array}$ \\
\hline$C$, core dia. $(\mathrm{nm})$ & 330 & 330 & 330 & 330 & \\
\hline$S$, shell thickness (nm) & 0 & 25 & 50 & 100 & \\
\hline $\begin{array}{l}\text { Overall diameter } C+2 S \\
(\mathrm{~nm})\end{array}$ & 330 & 380 & 430 & 530 & \\
\hline $\begin{array}{l}d_{p} \text {, dia. of mesopores } \\
(\mathrm{nm})\end{array}$ & - & 3.9 & 3.9 & 3.9 & 3.6 \\
\hline$d_{p} / S$ & $\infty$ & 0.156 & 0.078 & 0.039 & \\
\hline BET surface area $\left(\mathrm{m}^{2} / \mathrm{g}\right)$ & 592 & 996 & 1097 & 1269 & 1120 \\
\hline $\begin{array}{l}\text { Pore volume from } \mathrm{N}_{2} \\
\text { sorption, }\left(\mathrm{cm}^{3} / \mathrm{g}\right)\end{array}$ & 0.94 & 1.10 & 1.25 & 1.25 & 1.41 \\
\hline $\begin{array}{l}\text { Volume of hollow cores } \\
\left(\mathrm{cm}^{3} / \mathrm{g}\right)\end{array}$ & 4.26 & 1.53 & 0.89 & 0.39 & \\
\hline $\begin{array}{l}\text { Total Void from } \mathrm{N}_{2} \text { PSD } \\
+ \text { calculated macro-core } \\
\text { volume }\left(\mathrm{cm}^{3} / \mathrm{g}\right)\end{array}$ & 5.20 & 2.63 & 2.14 & 1.64 & \\
\hline $\begin{array}{l}\text { Total Volume with solid } \\
\text { carbon included assuming } \\
\text { carbon density of } 1.79 \\
\mathrm{~g} / \mathrm{cm}^{3}\end{array}$ & 5.76 & 3.19 & 2.70 & 2.20 & \\
\hline $\begin{array}{l}\text { Fraction of Interstitial } \\
\text { Region }\end{array}$ & 0.26 & 0.26 & 0.26 & 0.26 & \\
\hline $\begin{array}{l}\text { Fraction of Shell Region } \\
0.74\left[1-C^{3} /(C+2 S)^{3}\right]\end{array}$ & 0 & 0.26 & 0.41 & 0.56 & \\
\hline $\begin{array}{l}\text { Fraction of Core Region, } \\
0.74 C^{3} /(C+2 S)^{3}\end{array}$ & 0.74 & 0.48 & 0.33 & 0.18 & \\
\hline
\end{tabular}

\section{Electrochemical Properties}

Cyclic voltammetries (CV) were performed to evaluate the 60 capacitance performance of the as-synthesized porous carbons at scan rates of $1 \mathrm{mV} \mathrm{s}^{-1}$ to $200 \mathrm{mV} \mathrm{s}^{-1}$. The typical CV curves of porous carbons at scan rates 5 and $200 \mathrm{mV} \mathrm{s}^{-1}$ are shown in Fig. 6(a) and Fig. 6(b), respectively. The specific capacitance values of all the porous carbons were calculated according to 65 the equation,

$$
C=\frac{1}{2 \Delta V \cdot v} \int|j| d E
$$

where, $\Delta V$ is the voltage window applied in CV testing, $v$ is the scan rate and $j$ is the current density. The obtained specific gravimetric capacitance is plotted against scan rates in Fig. 7. 70 At low scan rates, rectangular curves are seen in Fig. 6(a) indicating little ionic transport limitations and the gravimmetric specific capacitance in Fig. 7, correlates with surface area of the carbon with the decreasing order of CCS150 > C-CS80 > C-CS50 > C-CS0. At $1 \mathrm{mV} \mathrm{s}^{-1}$, C-CS150 
shows the highest capacitance of $>150 \mathrm{~F} \mathrm{~g}^{-1}$. At high scan rate of $200 \mathrm{mV} / \mathrm{s}$, the CV curves in Fig. 6(b) are less rectangular and more distorted at both ends of the electrochemical window. The approach to the voltage limits in the CV curves 5 still sees the current density in decreasing order from CCS150 to C-CS0. But during current reversal (discharge) from the voltage limits, a reverse trend is observed indicating ion transport limitation. Discharge from mesopores saturated with ions is slower for the structure with a thicker shell. In all the 10 structures, it can be assumed that the hollow cores serve as ion reservoir and macro-channels of transport.
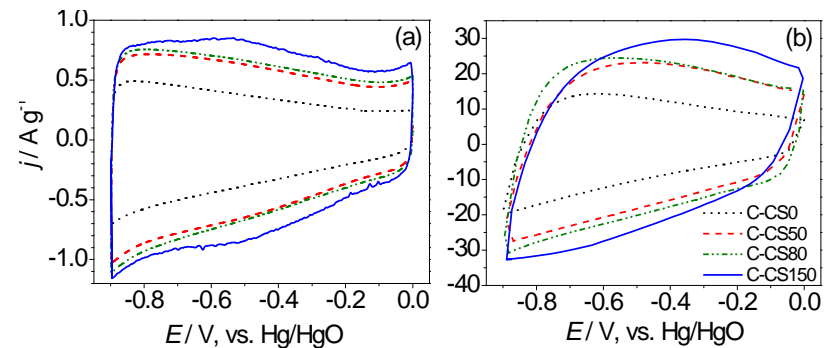

Fig. 6 CV curves of various carbons recorded at (a) $5 \mathrm{mV} / \mathrm{s}$ and (b) 200 15 $\mathrm{mV} / \mathrm{s}$.

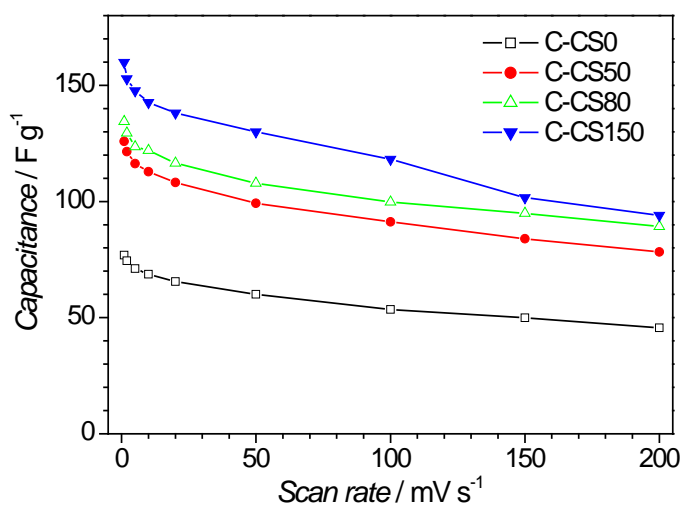

Fig. 7. Dependence of gravimetric specific capacitance on scan rates.

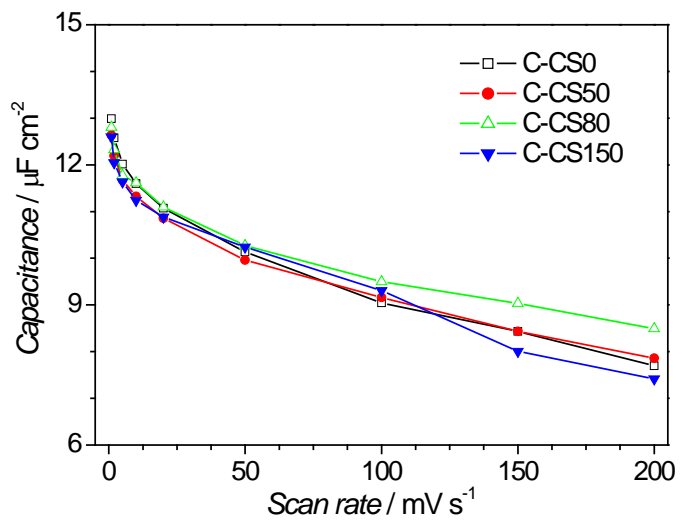

Fig. 8. Plot of specific capacitance per unit area verse scan rate.

20 The capacitance per mass is normalized to the surface area of each material in Fig. 8 to understand the effect of surface area of carbon structure on its capacitcance. The area specific capacitance values of C-CS0, C-CS50, C-CS80 and C-CS150 at lower scan rates are very similar. The chemical properties 25 of carbon surface in the porous carbons are identical as the materials are prepared under the same experimental conditions. Raman spectroscopy performed on the various synthesized carbon structures show the same ratio of $G$ band to $D$ band indicating the same ratio of graphitic to amorphous carbon 30 species. When the scan rate is increased, the specific capacitance per area of C-CS150 decreases more rapidly, and is even lower than other three kinds of porous carbons above $100 \mathrm{mV} \mathrm{s}^{-1}$. The core-shell carbon C-CS80 shows more steady capacitance with increasing scan rates and the best specific 35 capacitance at high scan rates. The assembly of high surface area mesoporous shells interconnected with hollow macrocores apears to offer the best structure with an optimal channel length for ion transport.

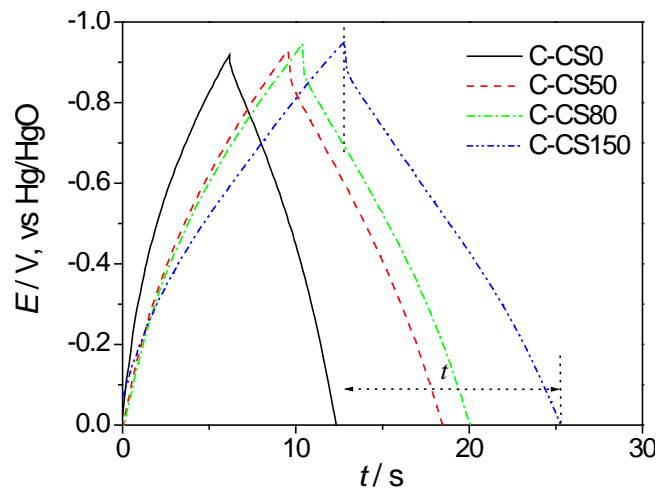

40

Fig. 9. Charge-discharge curves of C-CS0, C-CS50, C-CS80 and CCS150 obtained at a constant current density of $10 \mathrm{~A} \mathrm{~g}^{-1}$.

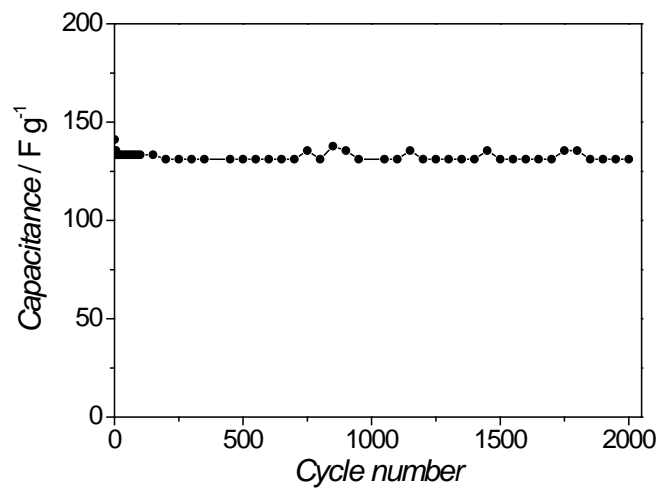

Fig. 10. Charge-discharge cycling stability of C-CS150 at a constant current density of $10 \mathrm{~A} / \mathrm{g}$.

45 The as-synthesized porous core-shell mesoporous carbons are further tested by charge/discharge cycles at various current loadings. The charge-discharge profiles of C-CS0, CCS50, C-CS80 and C-CS150 at a current density of $10 \mathrm{~A} \mathrm{~g}^{-1}$ were presented in Fig. 9. These curves are shown to be almost 50 symmetrical and an evident potential drop in the middle of each of the charge-discharge profiles is due to the ohmic drop. The discharge time, $\Delta t$ defined in Fig. 9, are estimated to be 6.2, 8.9, 9.7 and 12.2 s for C-CS0, C-CS50, C-CS80 and CCS150, respectively. Their corresponding capacitance is 55 calculated to be 68.9, 98.9, 107.8 and $135.6 \mathrm{~F} / \mathrm{g}$, respectively. In particular, C-CS150 has a very high capacitance retention over 2000 charge-discharge cycles as shown in Fig. 10. Also tested for comparison is the CMK-3 carbon templatesynthesized from SBA-15 silica. The Ragone plot of the as- 
synthesized porous carbons in Fig. 11 shows the relationship between the energy density and power density of the synthesized carbons. Each point is derived from the result of the charge/discharge curve of many cycles at a fixed current 5 loading between the voltage window of $0.9 \mathrm{~V}$ for a given porous carbon sample. Here, the energy density $(E)$ is calculated from the equation $E=C U^{2} / 4$ in a three-electrode system, where $C$ is the capacitance, and $U$ is the voltage window $0.9 \mathrm{~V}$ across the discharge profile. The power density ${ }_{10}(P)$ is then calculated from the $E$ using the equation $P=$ $E /(\Delta t)$, where $E$ is the energy density and $\Delta t$ is the discharge time. The energy density of the porous carbons C-CS0, CCS50, C-CS80 and C-CS150 gradually decreases with increasing power density in a trend similar to that in Fig. 7.

15 When high current loadings were applied, the energy density of C-CS150 decreased suddenly beyond a critical loading. This can be attributed to the apparent effect of a shell thickness of C-CS150 on ion transport in pores. An optimal capacitance performance can be obtained in C-CS80 with an 20 energy density of $5.9 \mathrm{Wh} \mathrm{kg}^{-1}$ and a power density of $11.2 \mathrm{~kW}$ $\mathrm{kg}^{-1}$. This value compared favorably to a previous result 6.1 Wh kg-1 and $4.5 \mathrm{~kW} \mathrm{~kg}^{-1}$ in an ordered mesoporous carbon having a larger surface area of $1304 \mathrm{~m}^{2} \mathrm{~g}^{-1}$ and longer channels channels inside. $^{12}$

25 The performance of CMK-3 carbon is also inferior to that of C-CS80 and C-CS150. The CMK-3 carbon has a small mesopore of $3.6 \mathrm{~nm}$ and no macropores. The typical particle size of a CMK-3 carbon powder is one to few micrometers. The CMK-3 curve in Fig. 11 drops early at about $10 \mathrm{~A} / \mathrm{g}$, 30 apparently due to the ionic transport resistance out of of the micron size powder. The better performance of the core-shell carbons is due to the optimium organisation of the hierarchical porous structure with hollow cores buffering ions in the mesoporous network.

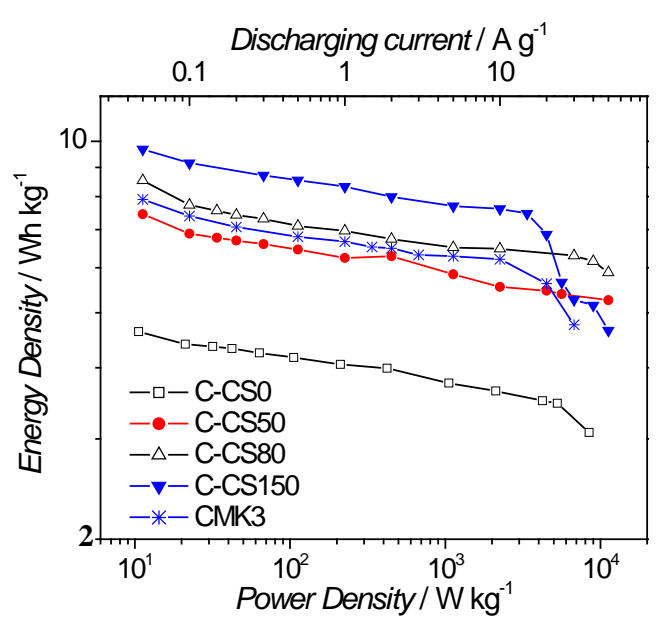

Fig. 11. Ragone plot of the synthesized porous carbons.

\section{Structure-Performance Relationship}

Stepwise variation of the thickness of mesoporous shell layer enables investigation of the structure-performance 40 relationship at a particular length scale and the relativity of impacts at different length scales. To better understand the results of the core-shell structures, we re-organize the family of curves in Fig. 7 and Fig. 11 to give a perspective on the quantitative effects of shell thickness. In Fig. 12, the changes 45 of structures and performance is plotted as a function of shell thickness. In an assembly of fcc spheres, the fraction of interstitial region (triangles in Fig. 12(a)) is 0.26 of the total volume regardless of the diameter of the spheres. The remaining fraction of 0.74 is shared by the shell and the core 50 regions. The relative amount of core region decreases and the shell region increases with shell thickness in the manner shown in Fig. 12(a). The most rapid structural change, however, is seen in the inverse aspect ratio of mesopore, in terms of $d_{p} / S$, which declines rapidly with increasing shell 55 thickness as shown in Fig. 12(a). Since the core size remains unchanged, the ratio of mesopore size to core diameter $d_{p} / C$ stays constant at 0.012 for all the carbon structures.
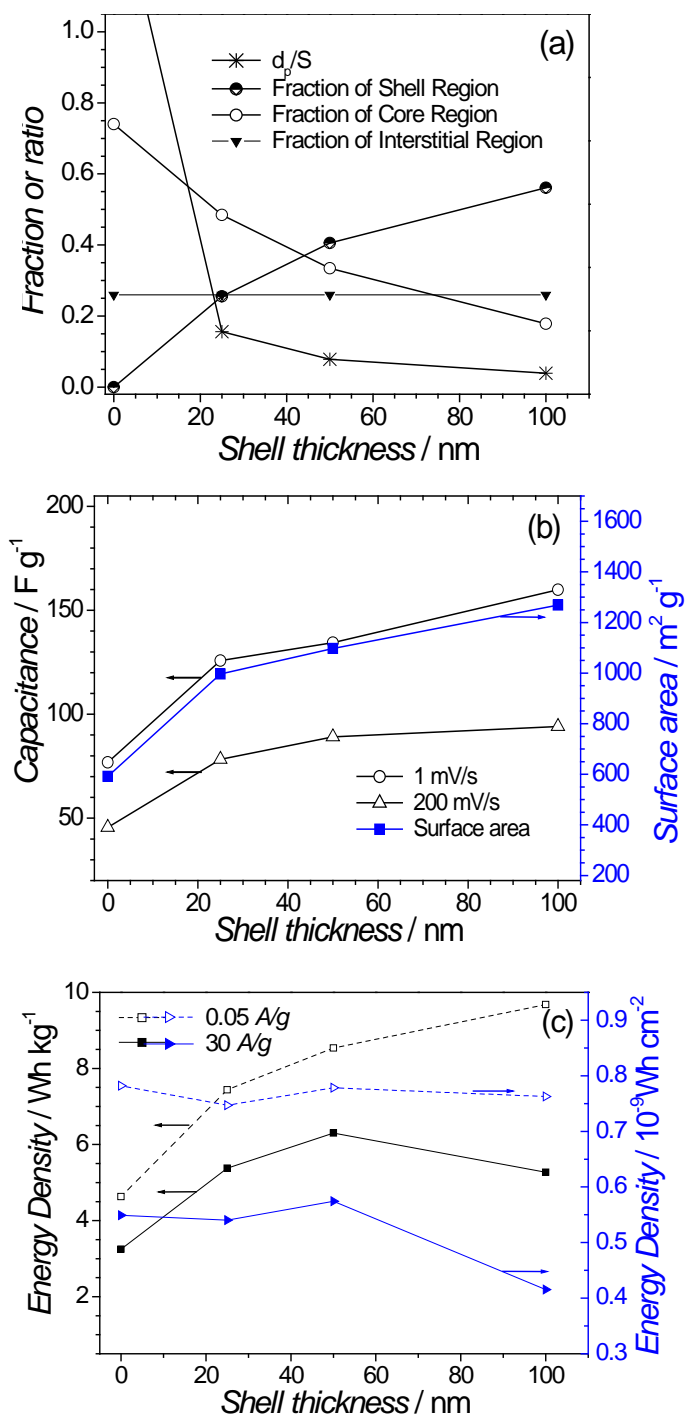

60

Fig. 12. (a) Variations in mesopore aspect ratio, $d_{p} / S$ and fractions of different regions in the hierarchical structure. (b) Correlations of specific gravimmetric capacitance to surface area (solid squares) at low scan rate (open circles) and high scan rates (open triangles).

65 (c) Gravimmetric energy density (triangles and right y-axis) and nitrogen area specific energy density (squares and left y-axis) at low current (open symbols connected by dashed line) and high current (solid symbols connected by solid lines). 
At low scan rates or low current loadings, ionic transport effects are small. Both capacitance and energy correlates directly with the total surface area of the hierarchy structure. This can be illustrated in Fig. 12(b) where capacitance is 5 plotted together with surface area as a function of shell thickness and a very good correlation can be seen. In Fig. 12(c), the energy density at low current increases monotonically (open squares) and can again be correlated directly to an increase of surface area. When normalized to 10 nitrogen surface area (open triangles), the energy delivered per unit nitrogen surface area is relatively constant with shell thickness.

At high scan rates or high current loadings, ionic transport becomes limiting in structures with a thick shell. The 15 appearance of an optimum shell thickness in the gravimmetric energy density curve (solid squares) can be related to comprimise of two factors: the surface area which increases monotonically with shell thickness and $d p / S$ which decreases monotonically. In Fig. 12(a), the $d_{p} / S$ curves decrease more

20 rapidly than the decrease in fraction of macro-cores. Given the macropore is orders of magnitude larger than mesopore. It is expected that transport in the mesopore to be limiting in the structure with a thick shell. In Fig. 12(b), the capacitance at $200 \mathrm{mV} / \mathrm{s}$ curve increases less than the expected surface area25 proportional amount. At high current loading of $30 \mathrm{~A} / \mathrm{g}$, the performance of the C-CS150 structure is lower than that of the C-CS80 structure, as shown in Fig. 12(c). The energy density per nitrogen surface area (solid triangles in Fig. 12(c)) is relatively constant among the first three structures C-CS0, C${ }_{30} \mathrm{CS} 50$, and C-CS80 but is reduced by half in the case of CCS150.

Through the set of core-shell porous structures, we understand better the behavior of electrochemical capacitance and ionic transport in a porous structure and the contribution 35 of porosities at different length scales. At low scan rates or low currents, all available surface area as determined by nitrogen sorption are fully utilized. The capacitance and energy are both proportional to surface area of the structure which increases monotonically in the series C-CSO to C${ }_{40} \mathrm{CS150}$. At high scan rate or high currents, ion-transport becomes limiting apparently in mesopores when pore diameter, $d_{p}$ is $1 / 25$ that of shell thickness, $S$. This study has been limited to variation of shell thickness when the core diameter, $C$, and mesopore diameter $d_{p}$ remains unchanged. 45 The synthesis described here allows changes of $C$ by using smaller silica spheres initially or changes in $d_{p}$ by different choices of surfactants. It will be of great interest to further investigate the relative effects of porosities at different length scales by synthesizing other sets of structures and evaluating 50 their electrochemical properties. These studies and data are also valuable to establishing a model predicting electrochemical performance from the geometric parameters of a hierachy structure.

\section{Conclusions}

55 Hierarchical core-shell porous carbons were successfully prepared by using core-shell silica spheres assemblies as templates. The as-synthesized ordered porous carbon structure has hollow macro-cores surrounded by mesoporous shells with interstitial space in between. A performance-structure 60 relationship can be established from the electrochemical tests on the set of hierarchical structures with stepwise increase in thickness of the mesoporous shell. At low scan rates and low currents, capcitance depends on surface area which increases with the mesoporous shell thickness. At high scan rates and 65 high current loadings, ion transport becomes limiting in thick shells. The carbon structure of C-CS80 has the best performance at very high currents when the structure appears to be optimum with sufficently thick shell of high surface area and the mesopore to shell thickness ratio, $d_{p} / S$ is not too low. 70 This structure with a hollow core of $330 \mathrm{~nm}$ and a shell thickness of $50 \mathrm{~nm}$, can deliver energy density of $5.9 \mathrm{Wh} \mathrm{kg}^{-1}$ at a power density of $11.2 \mathrm{~kW} \mathrm{~kg}^{-1}$.

\section{Acknowledgement}

Financial supports from GRF 7002/09P, NSFC/GRF [HKU 75 735/07] and University of Hong Kong SRT on Clean Energy Research are acknowledged. The SEM and TEM were performed in the Electron Microscopy Unit of University of Hong Kong. We also thank the Department of Physics for the use of their SEM.

\section{${ }_{80}$ Notes and references}

"Department of Chemistry, The University of Hong Kong, Pokfulam Road, Hong Kong SAR, P.R. China. E-mail: hrsccky@hku.hk; Fax: +852 28571586.

$\dagger$ Electronic Supplementary Information (ESI) available: The calculations 85 of structural parameters of the as-synthesized porous carbons. See DOI: $10.1039 / \mathrm{b} 000000 \mathrm{x} /$

1 D. Qu and H Shi, J. Power Sources, 1998, 74, 99-107.

2 C. Vix-Guterl, E. Frackowiak, K. Jurewicz, M. Friebe, J. Parmentier and F. Béguin, Carbon, 2005, 43, 1293-1302.

3 J. Chmiola, G. Yushin, Y. Gogotsi, C. Portet, P. Simon and P. L. Taberna, Science, 2006, 313, 1760-1763.

4 F. Beguin, Carbon, 2001, 39, 937-950.

5 A. Kajdos, A. Kvit, F. Jones, J. Jagiello and G. Yushin, J. Am. Chem. 95 Soc., 2010, 132, 3252-3253.

6 J. Lee, S. Han and T. Hyeon, J. Mater. Chem., 2004, 14, 478-486.

7 T. Bordjiba, M. Mohamedi and L. H. Dao, Adv. Mater., 2008, 20, 815-819.

8 I. Moriguchi, F. Nakahara, H. Yamada and T. Kudo, Solid-State Lett., 2004, 7, A221-A223.

9 H. Yamada, H. Nakamura, F. Nakahara, I. Moriguchi and T. Kudo, J. Phys. Chem. C., 2007, 111, 227-233.

10 D. Wang, F. Li, M. Liu, G. Lu and H. Cheng, Angew. Chem. Int. Ed. 2008, 47, 373-376.

10511 C. Portet, G.Yushin and Y.Gogotsi, J. Electrochem. Soc., 2008, 155, A531-A536.

12 F. J. Li, N. van der Laak, S. W. Ting and K. Y. Chan, Electrochim. Acta, 2010, 55, 2817-2823.

13 J. S. Yu, S. Kang, Y. B. Suk and G. Chai, J. Am. Chem. Soc., 2002, 110 124, 9382-9383.

14 G. S. Chai, S. B. Yoon, J. S. Yu, J. H. Choi and Y. E. Sung, J. Phys. Chem. B, 2004, 108, 7074-7079.

15 W. Stöber, A. Fink and E. Bohn, J. Colloid Interface Sci., 1968, 26, 62-69.

11516 J. H. Kim, S. B. Yoon, J. Y. Kim, Y. B. Chae and J. S. Yu, Colloids \& Surfaces A: Physicochem. Eng. Aspects, 2008, 313-314, 77-81.

17 C. L Burket, R. Rajagopalan, A. Marencic, K. Dronvajjala and H. C. Foley, Carbon, 2006, 44, 2957-2963. 